\title{
ANTECEDENTES Y MARCO CONCEPTUAL PARA UNA GESTIÓN SOSTENIBLE DE LA CALIDAD DEL AGUA EN LA CUENCA DEL RÍO BUENO, X REGIÓN DE LOS LAGOS. CHILE.
}

\section{BACKGROUND AND CONCEPTUAL FRAMEWORK FOR SUSTAINABLE MANAGEMENT OF WATER QUALITY IN THE RIO BUENO BASIN, X LAGOS REGION, CHILE.}

Sara Zelada Muñoz

Magíster en Ciencias, Mención Entomología. Dra. en Comunicación. Consultora independiente para evaluación de calidad biológica de aguas continentales utilizando macroinvertebrados como indicadores. E-mail sarazel@gmail.com

Recibido: 07 de Julio de 2017; Aceptado: 04 de Septiembre de 2017.

RESUMEN

El presente artículo hace una revisión de los trabajos publicados más recientes en relación a la gestión de los recursos hídricos en Chile bajo el concepto de calidad ecológica, resumiendo información para la gestión sostenible del agua en la cuenca hidrográfica del río Bueno de la Décima Región de Chile, donde se ha conformado la primera Mesa Provincial del Agua en la ciudad de Osorno (julio 2015). Los Derechos de Agua regulados por el Código de Aguas de 1981 y un déficit en la planificación territorial integrada, son los principales obstáculos para una gestión eficiente del recurso hídrico. Se hace un aporte en relación a los protocolos rápidos que se aplican a la evaluación de la calidad biológica del agua con macroinvertebrados bentónicos como indicadores, que son ampliamente utilizados en el mundo.

Palabras clave: estado ecológico, cuenca del río Bueno, gestión de recursos hídricos, macroinvertebrados.

\section{ABSTRACT}

This article reviews the most recent published works related to the management of water resources in Chile under the concept of ecological quality, summarizing information for the sustainable management of water in the river basin of the Rio Bueno of the Tenth Region of Chile, where the first Provincial Water Board has been formed in the city of Osorno (July 2015). The Water Rights regulated by the Water Code of 1981 and a deficit in integrated territorial planning are the main obstacles to an efficient management of water resources. A contribution is made in relation to the rapid protocols that are applied to the evaluation of the biological quality of the water with benthic macroinvertebrates as indicators, that are widely used in the world.

Key words: ecological status, basin of the river Bueno, management of water resources, macroinvertebrates. 


\section{INTRODUCCIÓN}

La calidad de las aguas se ha convertido en un asunto prioritario para la gestión de los recursos hídricos a nivel mundial. A principios de los años 90 se inicia en Europa un proceso de gestión integrada de los recursos de agua conducente a la dictación de la Directiva Marco sobre el Agua (DMA) que entra en vigor el año 2000, cuyos principales objetivos medioambientales consisten en lograr el mejor estado ecológico y estado químico posible para las aguas superficiales. La DMA propone realizar una tipología y una clasificación de las masas de agua y definir unas condiciones de referencia para cada tipo ecológico (DMA 2000, Martínez et al. 2004). Estas condiciones de referencia son cruciales para todo el proceso, constituyéndose en la base para los objetivos de protección del agua.

Para la DMA la calidad de los ecosistemas dulceacuícolas se evalúa tomando en consideración el estudio no sólo de los parámetros físico-químicos (temperatura, caudal, nutrientes, oxígeno, etc.) e hidrológicos, sino también aquellos parámetros bióticos como son la flora acuática (fitoplancton, macrófitos y fitobentos), así como los invertebrados bentónicos y peces como indicadores de calidad ecológica del sistema, y como sus componentes principales, considerándose como apoyo los datos hidromorfológicos y físico-químicos (DMA 2000, Martínez et al. 2004).

Parra (2009) sostiene que el agua es un recurso estratégico en un mundo que está cambiando de manera muy rápida, el agua dulce, en particular, no sólo estructura el paisaje y es la base del clima, sino que tiene una gran influencia en los patrones de crecimiento económico y demográfico, y advierte que el sostenido crecimiento económico de Chile en el último tiempo ha generado demandas hidroeléctricas, agrícolas y forestales, de riego, uso doméstico e industrial, acuicultura, requerimientos indígenas y otros, cuyos efectos colaterales indeseados como lo es la contaminación de las aguas, la pérdida de biodiversidad y disminución de los recursos naturales, suelen transformarse en conflictos con repercusiones económicas, políticas y ambientales.

Los conflictos arriba mencionados derivan, en gran parte, del marco legal e institucional que rige los derechos de aprovechamiento de las aguas (DAA) en Chile, regulados por el Código de Aguas (CA) de 1981 el cual establece que éstos son asignados a privados, que se transan libremente en el mercado y están altamente concentrados. Un segundo factor es la compartimentación existente de los numerosos organismos del Estado con responsabilidad en el ámbito de los recursos hídricos ( $R \mathrm{RHH}$ ), generando falta de transparencia por fragmentación de la información, cruzamiento de ésta y asignación difusa de responsabilidades, lo que se traduce en un déficit en la planificación territorial integrada. Por último, existe una ausencia total de mecanismos de resolución de conflictos dentro de las cuencas, debiendo llegar estos a la vía judicial (Meza 2011, Banco Mundial 2011, 2013).

La Ley N 19.300 sobre Bases Generales del Medio Ambiente del año 1994 logró institucionalizar la temática ambiental, en el país e incentivar en la población un mayor 
respeto por la preservación y conservación del medio ambiente, abriendo espacios de discusión y de participación ciudadana. A partir de las recomendaciones que la OCDE (2005) hace a Chile en cuanto a aumentar el tratamiento eficaz de efluentes industriales, se han ido incorporando normas de emisión para la mejora continua del marco regulatorio, sin embargo, aunque a la fecha más del $80 \%$ de las áreas protegidas tienen planes de manejo, estos están incompletos, sólo parcialmente implementados y desactualizados (OCDE 2016),

Los instrumentos que más se han utilizado hasta ahora en la gestión del agua en Chile son el Sistema de Evaluación de Impacto Ambiental (SEIA) que entró en vigencia en abril de 1997, y las normas de emisión y calidad del Ministerio del Medio Ambiente (MMA 2011). En 2005, por medio de la ley $N^{\circ} 20.017$, se realizaron importantes modificaciones al CA, constituyéndose la figura de caudal ecológico mínimo y de acuerdo al artículo 129, la Dirección General de Aguas (DGA) deberá velar por la preservación de la naturaleza y la protección del medio ambiente, considerando "las condiciones naturales pertinentes para cada fuente superficial".

El documento del Banco Mundial (BM 2011) proporciona un diagnóstico de la gestión de los recursos hídricos en Chile, señalando como prioritarios, en primer término, la protección de los DAA de grupos vulnerables, incluyendo los grupos indígenas y pequeños agricultores, en que una cantidad importante no tiene títulos o no están inscritos en los registros de los Conservadores de Bienes Raíces, y mejorar la protección de los requerimientos hídricos para los ecosistemas y servicios asociados. Entre los desafíos respecto a la institucionalidad, recomienda fortalecer la Dirección General de Aguas (DGA) y las organizaciones de usuarios como son las Juntas de Vigilancia (JdV) creadas en las cuencas, las que no están registradas en el Archivo Público de la DGA al menos en un 50\%, y muchas de ellas poseen limitada organización, capacitación y profesionalización.

\section{LA GESTIÓN DE CUENCAS EN CHILE.}

La cuenca contiene los recursos naturales (agua, suelo, vegetación, fauna) que interactúan con el aprovechamiento y los procesos productivos de los seres humanos. Los estudios actuales y las políticas territoriales se enfocan a la gestión de la cuenca hidrográfica definida como "la unidad territorial formada por un río (lago o laguna) con sus efluentes y por un área colectora de las aguas (el área de drenaje)" (Parra 2009), siendo considerada un ecosistema donde se integran o entrelazan tanto componentes naturales como elementos antrópicos (socioeconómicos y jurídico -institucionales) y su gestión requiere ser integral. El recurso hídrico fluye desde la cabecera de la cuenca hasta el mar u otro cuerpo de agua, y lo que ocurre en cualquier punto de su estructura afecta o condiciona el uso y calidad aguas abajo. Por esto, el enfoque de cuenca apunta al uso racional y efectivo del agua, que necesariamente pasa por la gestión local, involucrando a los usuarios del recurso, las autoridades, las instituciones, siendo de gran importancia "el ordenamiento y la planificación territorial desde la perspectiva de armonizar los componentes socio-cultural, económicos y ambientales" (SUBDERE 2013).

Dourojeani et al. (2002) señalan que "la gestión integrada del agua ha sido enfatizada y recomendada en todas las grandes conferencias internacionales sobre los 
recursos hídricos", y hacen mención del Programa 21 aprobado en la Conferencia de las Naciones Unidas sobre el Medio Ambiente y el Desarrollo (Río De Janeiro, 1992), la Directiva Marco Europea (Directiva 2000/60 CE), así como la Conferencia Internacional sobre el Agua Dulce (Bonn, Alemania, 2001) en donde se subraya el concepto de ordenación integrada de los recursos hídricos, incluyendo "la integración de los aspectos relativos a las tierras y a las aguas" que reconocen la cuenca o subcuenca. como base de una política de aguas, en cuanto a que "las características físicas del agua generan un grado extremadamente alto, y en muchos casos imprevisible, de interrelación e interdependencia (externalidades o efectos externos) entre los usos y usuarios de agua en una cuenca". La gestión del agua a nivel de cuencas ha sido recomendada repetidas veces por la OCDE (Akhmouch 2012, OCDE 2011, 2016).

El concepto de Gestión Integrada de los recursos Hídricos (GIRH) es diferente al de Cuencas. La Gestión de Cuencas incorpora entre otros: la protección de las características hidrológicas de la cuenca, control de la descarga de agua y de escorrentía superficial y subterránea, recuperación de tierras degradadas, control de la erosión, apoyo a la conservación de recursos y producción agropecuaria, apoyo al mejoramiento de la calidad de vida de habitantes y gestión ambiental. En tanto que la GIRH es un concepto más amplio, definido por la Global Water Partnership, como: "La GIRH es un proceso que promueve el desarrollo y gestión coordinada del agua, la tierra y los resultados relacionados, con el objetivo de maximizar el bienestar económico y social resultante de una manera equitativa sin comprometer la sustentabilidad de los ecosistemas vitales" (U de Chile 2001).

Los enfoques de gestión de GIRH, como sucede con los de manejo de cuenca, se pueden clasificar en varios tramos, dependiendo de la cantidad de temas que se incluyen en el proceso de gestión y de su complejidad: elaboración de estudios, administración del agua, construcción de obras hidráulicas, ejecución de proyectos, gestión integrada de los recursos hídricos (que integra a todas las demás y considera lo social, ambiental y económico, así como el control de las externalidades negativas de usuarios sobre los demás), apoyo a actividades de manejo de cuencas, entre otros (Dourojeanni 2010a).

Las Organizaciones de Usuarios del Agua (OUA) representan a los titulares de derechos de aprovechamiento de agua (DAA) y ellas son las Juntas de vigilancia (JV), las Comunidades de aguas subterráneas y los Comités de agua potable. Estos ejercen una función pública y están conformados por particulares que son titulares de los derechos de agua. Las JV ejercen la función de administradores del agua que es una función pública, pero acotada a la cuenca u hoya hidrográfica, labor que comparten con la Dirección General de Aguas (DGA) el máximo organismo que posee "las potestades y atribuciones regulatorias de las aguas es la DGA que es un órgano técnico de la administración del Estado, relevante en materia de aguas, depende directamente del Ministerio de Obras Públicas" (Vergara 2011).

Tanto las Comunidades de aguas como las Asociaciones de Canalistas se diferencian de las JV por su naturaleza privada. Uno de los problemas más serios que se suscitan es el de la resolución de conflictos, ya que las organizaciones de usuarios son allí juez y parte, lo que genera conflictos de intereses. Vergara (2011 op. cit.) considera que "para resolver esta situación es necesaria la creación de tribunales especializados capaces de tomar decisiones con independencia a los intereses individuales de los miembros que conforman las organizaciones de usuarios". 
Todas las organizaciones arriba mencionadas son reguladas por el Código de Aguas. "Sin embargo, otros problemas que afectan a las cuencas, tales como sequías, contaminación, erosión, etc., carecen de una institucionalidad capaz de responder eficazmente los desafíos que representan" (Dourojeanni, 2010b).

LAS MESAS DEL AGUA.

Como una alternativa para establecer acuerdos entre los usuarios, nacen las Mesas del Agua, que son un aporte a la solución de conflictos, con una visión de largo plazo y cuyos integrantes tienen un rol y una responsabilidad. Una mesa de agua es de este modo, una instancia donde se reúnen diferentes actores (gobierno, municipio, usuarios del agua, representantes de organizaciones, etc.) para conocerse, intercambiar puntos de vista, coordinar acciones, hacer propuestas, solucionar conflictos generados a partir del uso del agua y producir mecanismos para abordarlos.

Dourogeanni (2009), experto senior en recursos hídricos de Fundación Chile y ex director de Recursos Hídricos de Cepal, advierte que las mesas de diálogo creadas para coordinar acciones y minimizar conflictos, son opciones "buenas y útiles en la medida que se apliquen en forma pensada y ordenada y sin que falten los elementos esenciales, tales como respaldo legal y financiero para que las organizaciones de recursos hídricos por cuencas puedan cumplir sus roles". Sin embargo, la capacidad de gobernabilidad de la gestión de cuencas tropieza con el sistema político-administrativo, porque estos espacios están delimitados por razones naturales y no coinciden con dichos límites. Por su parte, los gestores de recursos naturales desconocen los límites y el funcionamiento de estos ecosistemas y falta apoyo del Estado. Por lo que considera necesario crear estas capacidades, resultando prioritarios los siguientes aspectos: 1) descentralizar la gestión del agua y darles más poder de decisión a los actores locales; 2) legislar apropiadamente para establecer las organizaciones de recursos hídricos por cuenca con su respectiva agencia; y 3) que los organismos de recursos hídricos por cuenca sean asistidos pero también fiscalizados por una entidad de nivel nacional.

Las Mesas Regionales del Agua están conformadas por la DGA de cada región y las preside el Gobernador Regional. Estas instancias regionales se han creado para avanzar hacia un manejo integrado del agua, otorgando "mayor participación a los actores relevantes en las decisiones de política y planificación en el ámbito de los recursos hídricos a nivel de cuencas" para un desarrollo sostenible del territorio (U. deChile, 2001). Se han constituido a la fecha en Tarapacá, Copiapó (Región de Atacama), Coquimbo, Valparaíso, La Araucanía.

\section{LA CUENCA DEL RÍO BUENO.}

El distrito de Lagos Araucanos (LA) (Thomasson 1963), también conocido como Lagos Norpatagónicos o de la Eco-Región Valdiviana (Abell et al. 2008), está ubicado entre los $37^{\circ}$ y $42^{\circ} \mathrm{S}$ y los $71^{\circ}$ y $72^{\circ} \mathrm{W}$. Los LA tienen un origen glacial o tectónico glacial común, generado por la retirada de los hielos que cubrieron esta zona durante la última glaciación denominada Llanquihue ocurrida entre 14.000 y 15.000 AP (Batist et al. 2008). Los LA son monomícticos y se caracterizan por una destacada condición oligotrófica; con aguas muy transparentes, escasa producción de algas y bajas concentraciones de nutrientes, particularmente nitrógeno $(N)$ y fósforo $(P)$ (Soto y 
Campos 1995, Woelf et al. 2003, DGA 2011, 2014). Los LA también presentan bajas concentraciones de clorofila y diversidad de macroinvertebrados y peces (Figueroa 2003, Fuentealba et al. 2010, Jara et al. 2006, Valdovinos 2008, Vila y Pardo 2008), los que son considerados como un buen indicador del estatus trófico de un sistema acuático oligotrófico.

La cuenca del río Bueno se extiende desde la latitud $39^{\circ} 50^{\prime}$ hasta la latitud $31^{\circ} 05^{\prime}$ Sur y es la quinta cuenca de Chile en importancia considerando su tamaño de $15.367 \mathrm{~km}^{2}$. Administrativamente pertenece a la Región de Los Ríos, y es compartida con la Región de Los Lagos. El área abarca las provincias de Ranco, Llanquihue y Osorno, y en ella se destacan como elementos fisiográficos la Cordillera de Los Andes volcánica activa, la precordillera morrénica, los lagos de barrera morrénica, la depresión intermedia o llano, la Cordillera de la Costa y planicies litorales y fluviomarinas. (DGA 2004).

Presenta dos tipos climáticos, templado cálido lluvioso con influencia mediterránea (en el sector centro y bajo de la cuenca) y clima templado frío lluvioso con influencia mediterránea en el sector precordillerano. Existen numerosos lagos en ella, destacándose los lagos Ranco, Puyehue, Rupanco y Maihue, encadenados de este a oeste por ríos receptores y emisarios. En la cuenca se han identificado cuatro volcanes activos con influencia directa sobre ella (DGA, 2004 op. cit.). El mismo estudio entrega información sobre la fauna íctica y bentónica de la cuenca, así como de la flora terrestre y acuática presente.

La cuenca está compuesta principalmente por las subcuencas del río Rahue y afluentes del lago Ranco y del Bueno. El río Rahue se origina en el extremo occidental del lago Rupanco y lleva sus aguas al río Bueno. Son afluentes del Rahue los ríos Forrahue, Negro y Damas. Afluentes del lago Ranco son los ríos Calcurrupe y Nilahue, que nacen el primero en el lago Maihue y el segundo en la cordillera de Los Andes. El río Bueno, por su parte, nace en el lago Ranco y va a desembocar en el océano Pacífico, siendo el río Pilmaiquén su principal afluente cuyo origen es el lago Puyehue. Una central hidroeléctrica se encuentra en funciones en el Pilmaiquén. a unos $36 \mathrm{~km}$ al Este de la ciudad de Osorno.

El lago Ranco se encuentra en la Región de Los Ríos, está situado a 70 m s.n.m., y posee una superficie cercana a los $410 \mathrm{~km}^{2}$, y una profundidad superior a $80 \mathrm{~m}$. Al oriente está rodeado de altas montañas y presenta varias penínsulas, senos, golfos e islas, siendo la isla Guapi la de mayor importancia. Su principal tributario es el río Calcurrupe que desagua el lago Maihue ubicado más al oriente. Otros afluentes del Ranco son los ríos Nilahue y Caunahue (DGA 2004). Un estudio realizado por la Universidad de Chile (DGA 2011) caracteriza al lago Ranco como ambiente generalmente oligotrófico, pudiendo mantener dicho estado si no se aumenta la carga de nutrientes actual. En cuanto a sus afluentes (Nilahue, Caunahue, Calcurrupe, Pitreño, Iculpe, Quiman) los resultados indicaron un estado contaminado producido principalmente por las descargas de pisciculturas y otras fuentes. Su bajo nivel de trofía predominante se ha mantenido a largo plazo. (DGA 2014).

El lago Maihue perteneciente a la Región de Los Ríos, tiene una superficie de 46 $\mathrm{km}^{2}$. y recibe como afluentes a los ríos Blanco, Hueinahue y Pillanleufu. Para este lago los estudios de DGA (2011) determinaron que su estado generalmente es oligotrófico, 
con valores de clorofila altos, más propios de una mesotrofía, previéndose que la oligotrofia se mantenga si no se aumenta la carga actual, proveniente principalmente de uso de suelo y poblaciones aledañas, dado que los valores de concentración de Fósforo medidos en los periodos de otoño, invierno y primavera, podrían generan un aumento de la condición trófica hacia niveles mesotróficos. Por lo tanto, DGA (2014) recomienda realizar control de descargas de Fósforo como por ejemplo, vertidos domiciliarios e industriales principalmente en periodo invernal, cuando el Fósforo limita el proceso de producción biológica. Los niveles bajos de trofía se han mantenido en el largo plazo.

El lago Puyehue perteneciente a la Región de Los Lagos, está situado a 212 m s.n.m., tiene una profundidad máxima de $135 \mathrm{~m}$. y un espejo de agua de $157 \mathrm{~km} 2$. Posee islas y una ensenada que es la bahía de Futacullín. Recibe como tributario el río Golgol, que desagua el lago Constancia, situado casi en la línea limítrofe. Otro río que drena el sector oriente y que vacía sus aguas en el lago Puyehue es el Chanleife, que tiene sus nacientes en el Volcán Casablanca (DGA, 2004). El lago mostró bajos niveles de trofía a través de todo un ciclo anual (2012-2013). Las concentraciones de Nitrógeno fueron bajas, lo que conllevaría a mantener niveles oligotróficos en este sistema. Por el contrario, los niveles de Fósforo fueron suficientemente altos como para generar episodios de mayor producción biológica y alcanzar una condición de Mesotrofía, por lo que "se deben implementar medidas de control de Nitrógeno por descargas superficiales de vertidos domiciliarios y vertidos industriales durante los periodos primavera, verano y otoño, sin embargo también se deben mantener descargas bajas de Fósforo. Estos registros deben complementarse con datos a más largo plazo. (DGA 2014).

El lago Rupanco pertenece administrativamente a la Región de Los Lagos, Provincia de Osorno, su cuenca se encuentra inmersa en los bosques templados lluviosos del sur de Chile, y constituye un corredor biológico y una zona de amortización para los Parques Nacionales de Puyehue y Vicente Pérez Rosales

De acuerdo a estudios de la DGA (2014 op. cit.), el lago Rupanco mostró una condición de Oligotrofia en el $100 \%$ de los casos estudiados durante los años 2012 y 2013 . La concentración de Nitrógeno es baja durante la primavera, lo cual permite mantener sus niveles de oligotrofía. Se recomienda "que para realizar manejo de condición trófica en el lago Rupanco se deben implementar medidas de control de Nitrógeno por descargas superficiales de vertidos domiciliarios y vertidos industriales al menos durante primavera. Su condición es de oligotrofia, e incluso de ultraoligotrofía según estudios de los años 2012 y 2013 y se acuerdo al análisis de concentración de nitrógeno (N) inorgánico presentaría "una baja potencialidad de aumentar el nivel trófico".

Factores incidentes en la Calidad del Agua en la Cuenca del Río Bueno son: la contaminación difusa por aguas servidas, contaminación difusa por ganadería, contaminación difusa por plaguicidas y fertilizantes y la descarga de RILES (DGA, 2004). El Informe PLADECO 2008- 2012 (CEDER, 2008) señala que los dos principales ríos (Damas y Rahue) debido a su constante y creciente contaminación, contribuyen a los problemas ambientales de la comuna de Osorno con las descargas de aguas servidas sin ningún tipo de tratamiento, el exterminio del bosque nativo, pérdidas de suelos por los desbordes de sus cauces y residuos sólidos urbanos.

Un estudio de la DGA (2012) de las cuencas de los ríos Valdivia y Rio Bueno entrega el catastro de puntos de agua (pozos, norias, bocatomas, centrales hidroeléctricas) superficiales y subterráneos. Además ha construido una Base de Datos 
(BDD) referencial para mejorar la consulta de estos datos y realizado una recopilación de los antecedentes disponibles de la zona, acudiendo a fuentes diversas en el territorio nacional. El mismo estudio ha constatado que el conocimiento de las aguas subterráneas no ha tenido un desarrollo relevante, en razón de que la fuente que sustenta el desarrollo regional han sido las aguas superficiales y los estudios que se han hecho son muy locales y puntuales, no existiendo una red de monitoreo de las aguas subterráneas.

\section{MACROINVERTEBRADOS BENTÓNICOS COMO BIOINDICADORES DE LA CALIDAD DEL AGUA.}

Se considera que un cuerpo de agua tiene una buena calidad biológica cuando posee características naturales que permiten el desarrollo en ella de las comunidades de organismos que le son propias. En Chile, los parámetros utilizados para la evaluación de la calidad de las aguas son de carácter físico- químico y, preferentemente, basados en la composición química. Los biocriterios han sido sugeridos sólo como herramienta complementaria recientemente (CONAMA 2004) y actualmente, no existe información sobre la calidad biológica del agua en Chile, salvo estudios científicos específicos (MMA 2011), con un número importante de estudios limnológicos que han caracterizado a los Lagos Araucanos aportando información biológica, basada en la concentración y diversidad del fitoplancton y zooplancton (De los Ríos y Soto 2002 y 2009, De los RíosEscalante 2008, DGA 2011, 2012, 2014, Woelfl et al. 2003) y algunos estudios de comunidades bentónicas (Figueroa et al. 2003, 2007, Parra et al. 2003, De los RíosEscalante et al. 2007, 2011, 2016,).

Ya en 1996, Alba-Tercedor señalaba que son miles los productos catalogados como altamente contaminantes, y muy pocos los que son analizados de manera sistemática. En la vigilancia y control de la contaminación en base a organismos bioindicadores existen multitud de metodologías que utilizan una amplia variedad de organismos: bacterias, protozoos, algas, macrófitos, macroinvertebrados, peces. Indicando que variaciones inesperadas en la composición y estructura de las comunidades de organismos vivos de los ríos pueden interpretarse como signos evidentes de contaminación. De todas las metodologías, aquellas basadas en el estudio de los macroinvertebrados acuáticos son las mayoritarias.

Los métodos de evaluación de la calidad de las aguas basados en macroinvertebrados son sencillos y rápidos, exigen escaso nivel de conocimientos previos y sus resultados son fiables, esto los hace idóneos para la vigilancia rutinaria de las cuencas fluviales, es así como "un operario puede evaluar y cartografiar muchos puntos diferentes en un solo día, con el único gasto inherente al desplazamiento", Luego en un mapa cartográfico el responsable de la gestión ambiental "puede en un golpe de vista observar los puntos conflictivos, y aquellos por el contrario presentan una calidad muy buena, buena o aceptable" .Estas metodologías no constituyen una novedad, datan de principios del siglo XX (Alba-Tercedor, 1996 op. cit.).

Martínez et al. (2004) señalan que en España se han realizado importantes trabajos para la determinación del estado ecológico de los ecosistemas acuáticos en grandes cuencas fluviales nombrando a autores que estudian el Duero, el Ebro, los ríos catalanes y del País Vasco. Afirman que estudios en la red hidrográfica del Júcar datan de 1993, y fueron ellos los primeros en utilizar los macroinvertebrados y otros índices biológicos para establecer la calidad del agua. 
El índice British Monitoring Working Party (BMWP) que utiliza macroinvertebrados como bioindicadores ha sido adaptado para Chile por Figueroa et al. $(2003,2007)$ como Indice Biótico de Familias de Chile (ChIBF). Estos investigadores sugieren que es un buen indicador de la calidad de las aguas de los ríos de cuencas agrícolas y ganaderas del sur de nuestro país y sólo requiere identificar los macroinvertebrados a nivel de familia, lo que significa que puede ser utilizado por personal sin gran experiencia taxonómica. El valor de ChIBF obtenido se hace corresponder con una determinada clase de calidad cotejándolo con la tabla, donde se califica la calidad en un rango que va de "muy buena" a "muy mala".

En Latinoamérica los índices con macroinvertebrados como indicadores se han aplicado a sistemas lóticos en países como Argentina, Colombia, Brasil, Nicaragua, México y en Chile (Gamboa et al. 2008). Sus ventajas costo-efectivas son evidentes, el resultado es que aporta información integrada del ecosistema y es capaz de cubrir una amplia región biogeográfica monitoreando sitios potencialmente impactados, facilitando su control y así es posible focalizar los esfuerzos de control y rehabilitación. Por ejemplo, el estudio de Figueroa et al. (2003) en río Damas (X Región de Los Lagos) permitió identificar una elevada diversidad de macroinvertebrados con respecto a otras cuencas, y detectar una gradiente desde la cabecera de la cuenca hasta su confluencia con el río Rahue, revelando que la riqueza de taxa disminuye, lo que se atribuyó a los fertilizantes empleados en las praderas de ganadería.

Las ventajas de utilizar macroinvertebrados como indicadores de calidad del agua han sido destacadas por DGA (2010): son uno de los componentes más abundantes de la comunidad bentónica, por lo que serían adecuados para realizar monitoreos biológicos de calidad de agua; su tolerancia a diferentes calidades del agua ha sido ampliamente estudiada, sobre todo en el hemisferio norte; por la diversidad de formas y hábitats que cubren; los hábitat de los macroinvertebrados bentónicos son muy variados y a cada uno de ellos corresponde una comunidad; son sedentarios y de larga vida, por lo tanto pueden revelar la calidad del agua en el punto de muestreo y ser extrapolables en el tiempo.

CONAMA (2010) señala que uno de los Indicadores de contaminación es la materia orgánica (MO) y que altas concentraciones se han correlacionado adecuadamente con los macroinvertebrados bentónicos, habiéndose generalizado su uso $y$, en especial los insectos, como indicadores de calidad del agua en todo el mundo. Hace además una relación de estos índices desde el de Hilsenhoff (1988), hasta el ChIBF aplicado en Chile por Figueroa et. al (2003), y declara como objetivo introducir en Chile la bioindicación en la evaluación y fiscalización de la calidad del agua, con la ventaja de ser un método comparable a otros países (Figueroa et al. 2007).

En Chile ya se han iniciado experiencias mediante el uso de estos bioindicadores con índices bióticos para ríos de diferentes zonas hidrológicas. Han sido aplicados con mayor frecuencia en la zona subhúmeda y húmeda (CENMA, 2010, Figueroa et al, 2003, Figueroa et al., 2007, Parra et al. 2003). Para lagos del Hemisferio Norte se han desarrollado, recomendado y aplicado índices unimétricos y multimétricos que incorporan macroinvertebrados bentónicos como indicadores (EPA 1998, Revenga et al. 2005, Solimini et al. 2006, Vernaux et al. 2004). En Estados Unidos una guía técnica para biocriterios ha sido desarrollada por la Environmental Protection Agency (EPA 1990) 
y una propuesta de biocriterios y metodologías para la implementación y monitoreo de la Norma Secundaria de Calidad Ambiental ha sido realizada por DGA (2010) para Chile.

\section{CONCLUSIONES.}

La gestión de los recursos hídricos en Chile recién comienza a implementarse y es importante revisar la información existente (publicaciones, estudios, informes, normativas y legislación) con el propósito de modelar un perfil de los conocimientos que se han ido generando, en parte debido a las presiones de la actividad antrópica sobre los cuerpos de agua dulce, y también por exigencias de la economía global, la incorporación de nuestro país a la OCDE, las instancias de gobernabilidad mundial del agua y del medio ambiente, fuertemente amenazados por la industrialización, la actividad minera, la reconversión de los suelos y urbanización.

La institucionalidad chilena para la gestión de las aguas es extremadamente compleja, compartida por diversos organismos sectoriales, con innumerables fallos en la administración, información, fiscalización, monitoreo, vigilancia y control, descoordinación, definición de roles, y ausencia de normas de calidad, en particular Normas Secundarias (BM, 2013).

La cuenca del Río Bueno ha sido estudiada parcialmente, existen informes actualizados para el Lago Ranco, sus afluentes y efluentes (DGA, 2011) consistentes en una evaluación crítica de estudios limnológicos anteriores, así como el monitoreo de la columna de agua y la capacidad crítica de nutrientes y fuentes de eutrofización, Incluye, además, una propuesta de monitoreo en el futuro, sugiriendo estaciones de muestreo, frecuencia estacional, monitoreo de fitoplancton, y un estudio de hidrodinámica del lago que no se ha hecho. En los ríos estudiados (Nilahue, Quimán, Pitreño) se observaron valores altos de $\mathrm{N}$ y $\mathrm{P}$ que indican contaminación, atribuible a descargas de las pisciculturas y otras fuentes.

En este estudio se hace un aporte dirigido a considerar los macroinvertebrados y el índice ChIBF como una herramienta útil para establecer el estado ecológico de los ríos de la Cuenca Hidrográfica del río Bueno, considerando que diversos estudios han detectado un avance hacia la mesotrofia en los cuerpos lacustres, debido a las descargas contaminantes, principalmente de nutrientes $(P, N)$ que afectan a sus ríos afluentes y mal manejo de las cuencas hidrográficas en el sur de Chile. Las herramientas de manejo actualmente disponibles podrán prevenir respuestas indeseadas, a veces irreversibles, frente a efectos combinados de eutrofización y cambio climático.

\section{REFERENCIAS}

Abell, R, M Thieme, C Revenga, C Bryer, 2008. Freshwater Ecoregions of the World: A New Map of Biogeographic Units for Freshwater Biodiversity Conservation. Bioscience 58: 403: 414

Alba-Tercedor, J. 1996. Macroinvertebrados acuáticos y calidad de las aguas de los ríos. IV Simposio del Agua en Andalucía (SIAGA) Almería, 1996, vol. II: 203-213 
Akhmouch, A. 2012, "Water Governance in Latin America and the Caribbean: A MultiLevel Approach", OECD Regional Development Working Papers, 2012/04, OECD Publishing. http://dx.doi.org/10.1787/5k9crzqk3ttj-en

Banco Mundial, 2011. CHILE. Diagnóstico de la gestión de los recursos hídricos

Departamento de Medio Ambiente y Desarrollo Sostenible. Región para América Latina y el Caribe 31 de marzo de 2011

Banco Mundial, 2013. Cbile. Estudio para el mejoramiento del marco institucional para la gestión del agua. Documento. Unidad de Ambiente y Aguas. Departamento de Desarrollo Sostenible. Región de América Latina y el Caribe.

CENMA (2010). Propuesta de utilización de biocriterios para la implementación y monitoreo de la Norma Secundaria de Calidad Ambiental. Criterios y metodologías. informe final

Centro de Estudios Regionales (CEDER), 2008. Informe 2. Diagnóstico Descriptivo General y Temático de la Comuna de Osorno. PLADECO 2008-2012

CONAMA, 2004. Guía CONAMA para el establecimiento de las normas secundarias de calidad ambiental para aguas continentales superficiales y marinas. Chile, CONAMA.

CONAMA, 2010. Clasificación de cuerpos de agua. ANEXOS. Departamento de Ciencias Ambientales y Recursos Naturales Renovables. Facultad de Ciencias Agronómicas Universidad de Chile Santiago, 31 de mayo de 2010

De los Ríos- Escalante, P. 2008, A null model for explain crustacean zooplankton species associations in Central and Southern Patagonian inland waters. An. Inst. Patagonia, 2008. 36: 25-34

De los Ríos- Escalante, P., 2017. Non randomness in spatial distribution in two inland water species malacostracans J. of King Saud Univ. Sci. 29, 260-262

De Los Ríos-Escalante, P, E Hauenstein, P Acevedo, X Jaque 2007. 1littoral Crustaceans in Mountain Lakes Of Huerquehue National Park (38॰S, Araucania Region, Chile) Crustaceana 80: 401-410

De los Ríos- Escalante, P, D Soto 2009. Estudios limnológicos en lagos y lagunas del Parque Nacional Torres Del Paine ( $\left.51^{\circ} \mathrm{S}, \mathrm{CHILE}\right)$. An. Inst. Patagonia, 2009. 37: 63-71

De los Ríos- Escalante, P, A Mansilla, C Anderson, 2011. The presence of the genus Hyalella (Smith, 1875) in water bodies near Puerto Williams (Cape Horn Biosphere Reserve, $54^{\circ} \mathrm{S}$, Chile) (Crustacea, Amphipoda). Pan-American Journal of Aquatic Sciences (2011), 6(4):273-279

De los Ríos- Escalante, P, A. Mansilla, 2017. Spatial patterns of Pisidium chilense (Mollusca Bivalvia) and Hyalella patagonica (Crustacea, Amphipoda) in an unpolluted stream in Navarino island (54_S, Cape Horn Biosphere Reserve). J. of King Saud Univ. Sci., 29: 28-31

DGA, 2004, Diagnóstico y Clasificación de los Cursos y Cuerpos de Agua según Objetivos de Calidad. Cuenca del Rio Bueno.Diciembre 2004

DGA, 2010. Propuesta de Utilización de Biocriterios para la Implementación y Monitoreo de la Norma Secundaria de Calidad Ambiental Criterios y Metodologías Informe Final. Gobierno de Chile Ministerio de Obras Públicas. Dirección General de Aguas 
Dpto. de Conservación y Protección de Rec. Hídricos. Realizado Por: Centro Nacional Del Medio Ambiente S.I.T. No 216 Tomo I De II. Santiago, Julio 2010.

DGA, 2011. "Antecedentes para Evaluar el Impacto Económico y Social de una Norma Secundaria de Calidad de Aguas en el Lago Ranco, Cuenca del Río Bueno". Informe Final Gobierno De Chile Ministerio De Obras Públicas Dirección General De Aguas. Realizado por $U$ de Chile.

DGA, 2012. Estudio cuencas principales Región de Los Rios. Informe final

Realizado por: Amphos 21 Consulting Chile Ltda. s.i.t. $n^{\circ} 293$.

DGA, 2014. Evaluación de la Condición Trófica de la Red de Control de Lagos de la DGA.

Realizado por M\&W Ambientales. Disponible en:

http://documentos.dga.cl/LG05518.pdf

DMA 2000/60/CE, 2000. Directiva Marco del Agua. Diario Oficial de las Comunidades Europeas. 22.12.2000

Dourojeanni A., 2009. Los Desafíos De La Gestión Integrada De Cuencas Y Recursos Hídricos En América Latina Y El Caribe. Fundación Chile, Santiago de Chile, Noviembre 2009

Dourojeanni A., 2010a. Diferencias conceptuales entre los términos "Manejo Integrado de Cuencas y "Gestión Integrada de los Recursos Hídricos").

http://www.academia.edu/5271223/DIFERENCIAS_CONCEPTUALES_ENTRE_LOS_T\%C 3\%89RMINOS (visitada 10 de abril 2017)

Dourojeanni A., 2010b. Las Mesas del Agua y la Gestión de Cuencas en Chile. Estudio de Caso. Región de Atacama, Chile. Fundación Chile.

https://research.csiro.au/gestionrapel/wp-content/uploads/sites/79/2016/12/Lasmesas-del-agua-y-la-gesti\%C3\%B3n-de-cuencas-en-Chile-2010.pdf (visitada 20 de mayo 2017)

Dourojeanni, A, A Jouravlev, G Chávez, 2002. Gestión del agua a nivel de cuencas: teoría y práctica. CEPAL - SERIE Recursos Naturales e Infraestructura $N^{\circ} 47$. Santiago de Chile, agosto de 2002

EPA, 1990. Biological Criteria. National Program Guidance forSurface Water, https://nepis.epa.gov/Exe/ZyPDF.cgi/000010KG.PDF?Dockey=000010KG.PDF (visitada el 2 de abril 2017)

EPA, 1998. Lake and reservoirs bioassessment and biocriteria. Tecnichal Guide Document.

http://monitoringprotocols.pbworks.com/f/Lake+and+Reservoir+Biocriteria+1998++200040DM.pdf (visitada el 10 de abril 2017)

Figueroa, R, C Valdovinos, E Araya, O Parra 2003. Macroinvertebrados bentónicos como indicadores de calidad de agua de ríos del sur de Chile. Rev. Chilena Hist. Nat. 76: 275-285.

Figueroa, R, A Palma, V Ruiz, X Niell, 2007. Análisis comparativo de índices bióticos utilizados en la evaluación de la calidad de las aguas en un río mediterráneo de Chile: río Chillán, VIII Región. Rev. Chilena Hist. Nat. 80: 225-242.

Fuentealba, C, R Figueroa, J Morrone, 2010. Análisis de endemismo de moluscos dulceacuícolas de Chile. Rev. Chilena Hist. Nat. 83: 289-298. 
Gamboa, M, R Reyes, J Arrivillaga, 2008. Macroinvertebrados bentónicos como bioindicadores de salud ambiental. Bol. Malar. Sal. Amb.. XLVIII, No 2, AgostoDiciembre.

Hilsenhoff, W. 1988. Rapid Field Assessment of Organic Pollution with a Family-Level Biotic J. North American Benthol. Soc., 7: 65-68

Martínez, J, E Correcher, A Piñón, M Martínez, A Pujante, 2004. Estudio del estado ecológico de los ríos de la cuenca hidrográfica del Júcar (España) mediante el índice BMWP' Limnetica 23: 331-346

Jara, C, 1989. Aegla denticulata-lacustris, New Subspecies, From Lake Rupanco, Chile. Proceed. Biol. Soc. Washington 102: 385-393.

Meza, R, J McPhee, 2011. Revisión del estado del arte en la gestión integrada de cuencas y sus lecciones respecto a la implementación de la estrategia nacional de gestión integrada de cuencas. XX Congreso Chileno de Hidráulica. Santiago, Chile

MMA, 2011. Informe del Estado del Medio Ambiente 2011, Cap. 8 Recursos Hídricos. Official Environment Status Report 2011: Environmental Heritage

MMA, 2013. Primer Reporte del Estado del Medio Ambiente. http://metadatos.mma.gob.cl/sinia/M2500MIN2013.pdf (visitada el 3 de abril 2017)

OCDE, 2005 Environmental Performance Review, Chile 2005 http://www.sinia.cl/1302/articles-57009_Chile_EDA2005_EMT2011x.pdf

OCDE, 2016. Environmental Performance Reviews (EPRs). Highlights Chile. http://oe.cd/epr. (Visitada el 2 de marzo 2017)

Parra O, 2009. Gestión Integrada De Cuencas Hidrográficas. (Documento Introductorio) Centro de Ciencias Ambientales, EULA-Chile Universidad de Concepción Junio 2009. http://www.eula.cl/doc/Cuencas\%20Hidrografica\%20Dr\%20Parra.pdf (visitada 8 de marzo 2017)

Parra O, C Valdovinos, R Urrutia, M Cisternas, E Habit, M Mardones, 2003. Caracterización y tendencias tróficas de cinco lagos costeros de Chile Central. Limnetica 22: 51-83.

Solimini AG, G Free, I Donohue, K Irvine, M Pusch, B Rossaro, L Sandin, AC Cardoso, 2006. Using

Benthic Macroinvertebrates to Assess Ecological Status of Lakes Current Knowledge and Way Forward to Support WFD Implementation. Institute for Environment and Sustainability. $47 \mathrm{pp}$

Soto, D, 2002. Oligotrophic patterns in southern Chilean lakes: the relevance of nutrients and mixing depth. Rev. Chilena Hist. Nat. 75:377-393.

SUBDERE, 2013. Gobierno de Chile. Guía Análisis y Zonificación de Cuencas Hidrográficas para el Ordenamiento Territorial. junio 2013. Disponible en http://www.subdere.gov.cl/sites/default/files/documentos/guia_zonificacion_fin al_con_isbn.pdf (visitada el 16 de abril 2017)

Thomasson K (1963) Auracanian lakes. Acta Phytogeog. Suecica 47: 1-139. 
Universidad De Chile, 2001. Estudio "Gestión Integrada de los Recursos Hídricos en Chile". Informe final. Facultad De Ciencias Agronómicas. Departamento De Ciencias Ambientales Y Recursos Naturales Renovables. Jefe de Proyecto Rodrigo Fuster

Valdovinos, C, 2008. Diversidad de Especies Invertebrados Dulceacuícolas. En Biodiversidad de Chile Patrimonio y desafíos. CONAMA, 2008. Segunda Edición, págs. 202-223.

Vergara, A, 2011. Administración y distribución de las aguas en Chile. Agronomía y Forestal 41: 10-13

Vila, I, R. Pardo, 2008. Diversidad De Especies Peces Límnicos. En CONAMA, 2008 Biodiversidad de Chile Patrimonio y desafíos. Diversidad De Especies

Verneaux V, J Verneaux, A Schmitt, C Lovy, \& JC Lambert, 2004a. The Lake Biotic Index (LBI): an applied method for assessing the biological quality of lakes using macrobenthos; the Lake Châlain (French Jura) as an example. Ann. Limnol. - Int. J. Lim. 40: 1-9. 